\title{
POWER-LAW TEMPLATE FOR INFRARED POINT-SOURCE CLUSTERING
}

\author{
Graeme E. Addison ${ }^{1}$, Joanna Dunkley ${ }^{1,2,3}$, Amir Hajian ${ }^{2,3,4}$, Marco Viero $^{5,6}$, J. Richard Bond ${ }^{4}$, Sudeep Das $^{2,3,7}$, \\ Mark J. Devlin ${ }^{8}$, Mark Halpern $^{9}$, Adam D. Hincks ${ }^{2}$, Renée HlozeK $^{3}$, Tobias A. Marriage ${ }^{3,10}$, Kavilan Moodley $^{11}$, \\ Lyman A. Page ${ }^{2}$, Erik D. Reese ${ }^{8}$, Douglas Scott ${ }^{9}$, David N. Spergel ${ }^{3}$, Suzanne T. Staggs ${ }^{2}$, And Edward Wollack $^{12}$ \\ ${ }^{1}$ Sub-department of Astrophysics, University of Oxford, Denys Wilkinson Building, Keble Road, Oxford OX1 3RH, UK \\ 2 Joseph Henry Laboratories of Physics, Jadwin Hall, Princeton University, Princeton, NJ 08544, USA \\ ${ }^{3}$ Department of Astrophysical Sciences, Peyton Hall, Princeton University, Princeton, NJ 08544, USA \\ ${ }^{4}$ Canadian Institute for Theoretical Astrophysics, University of Toronto, Toronto, ON M5S 3H8, Canada \\ ${ }^{5}$ Department of Astronomy, California Institute of Technology, 1200 East California Boulevard, Pasadena, CA 91125, USA \\ ${ }^{6}$ Department of Astronomy \& Astrophysics, University of Toronto, 50 St. George Street, Toronto, ON M5S 3H4, Canada \\ ${ }^{7}$ Berkeley Center for Cosmological Physics, LBL and Department of Physics, University of California, Berkeley, CA 94720, USA \\ ${ }^{8}$ Department of Physics and Astronomy, University of Pennsylvania, 209 South 33rd Street, Philadelphia, PA 19104, USA \\ ${ }^{9}$ Department of Physics and Astronomy, University of British Columbia, Vancouver, BC V6T 1Z4, Canada \\ ${ }^{10}$ Department of Physics and Astronomy, Johns Hopkins University, 3400 North Charles Street, Baltimore, MD 21218-2686, USA \\ ${ }^{11}$ Astrophysics and Cosmology Research Unit, School of Mathematical Sciences, University of KwaZulu-Natal, Durban 4041, South Africa \\ ${ }^{12}$ NASA/Goddard Space Flight Center, Code 665, Greenbelt, MD 20771, USA \\ Received 2011 August 23; accepted 2012 April 13; published 2012 June 4
}

\begin{abstract}
We perform a combined fit to angular power spectra of unresolved infrared (IR) point sources from the Planck satellite (at 217, 353, 545, and $857 \mathrm{GHz}$, over angular scales $100 \lesssim \ell \lesssim 2200$ ), the Balloon-borne Large-Aperture Submillimeter Telescope (BLAST; 250, 350, and $500 \mu \mathrm{m} ; 1000 \lesssim \ell \lesssim 9000$ ), and from correlating BLAST and Atacama Cosmology Telescope (ACT; 148 and $218 \mathrm{GHz}$ ) maps. We find that the clustered power over the range of angular scales and frequencies considered is well fitted by a simple power law of the form $C_{\ell}^{\text {clust }} \propto \ell^{-n}$ with $n=1.25 \pm 0.06$. While the IR sources are understood to lie at a range of redshifts, with a variety of dust properties, we find that the frequency dependence of the clustering power can be described by the square of a modified blackbody, $v^{\beta} B\left(v, T_{\text {eff }}\right)$, with a single emissivity index $\beta=2.20 \pm 0.07$ and effective temperature $T_{\text {eff }}=9.7 \mathrm{~K}$. Our predictions for the clustering amplitude are consistent with existing ACT and South Pole Telescope results at around 150 and $220 \mathrm{GHz}$, as is our prediction for the effective dust spectral index, which we find to be $\alpha_{150-220}=3.68 \pm 0.07$ between 150 and $220 \mathrm{GHz}$. Our constraints on the clustering shape and frequency dependence can be used to model the IR clustering as a contaminant in cosmic microwave background anisotropy measurements. The combined Planck and BLAST data also rule out a linear bias clustering model.
\end{abstract}

Key words: cosmic background radiation - cosmology: observations - infrared: diffuse background - infrared: galaxies - submillimeter: diffuse background

Online-only material: color figures

\section{INTRODUCTION}

The angular power spectrum of cosmic microwave background $(\mathrm{CMB})$ temperature fluctuations currently provides vital constraints on cosmological models (e.g., Komatsu et al. 2011; Larson et al. 2011). Experiments including the Atacama Cosmology Telescope (ACT), South Pole Telescope (SPT), and Planck satellite are now probing the CMB temperature power spectrum on arcminute scales (Das et al. 2011; Keisler et al. 2011; Planck Collaboration et al. 2011a). An improved measurement of the Silk damping tail (Silk 1968) improves constraints on, for instance, the scale dependence of primordial fluctuations, important for testing inflationary models, the number of relativistic species, and early-universe exotica (e.g., Komatsu et al. 2011). On arcminute scales the contribution to the angular power spectrum from the primary CMB fluctuations becomes subdominant to extragalactic foregrounds including infrared and radio point sources (e.g., White \& Majumdar 2004; Righi et al. 2008), and the thermal and kinetic Sunyaev-Zel'dovich effects (SZ; Sunyaev \& Zel'dovich 1970). Extracting the CMB signal requires understanding how the contribution from these components varies with frequency and angular scale.

The infrared (IR) point-source foreground is understood to originate from high-redshift $(z \sim 1-4)$ star-forming galaxies whose rest-frame emission peaks in the far-infrared due to thermal emission from dust grains illuminated by starlight (e.g., Bond et al. 1986, 1991; Hughes et al. 1998; Blain et al. 1999; Draine 2003). While our work concerns observations made in the millimeter and submillimeter we refer to these dusty sources throughout as "IR" sources. Thermal dust emission from starforming galaxies is also an important component of the cosmic infrared background (CIB — e.g., Puget et al. 1996). Galaxies trace the large-scale structure and so are clustered, with a scaledependent contribution to the power spectrum in addition to Poisson shot noise (Peebles 1980). Clustering of IR sources was therefore expected (e.g., Bond 1996, and references therein; Negrello et al. 2007) and has been detected in Spitzer Space Telescope data at $160 \mu \mathrm{m}$ (Lagache et al. 2007), by the Balloonborne Large-Aperture Submillimeter Telescope (BLAST) and Herschel Space Observatory at 250, 350, and $500 \mu \mathrm{m}$ (Viero et al. 2009; Cooray et al. 2010; Amblard et al. 2011), in the microwave sky at around 150 and $220 \mathrm{GHz}$ by SPT and ACT (Hall et al. 2010; Dunkley et al. 2011; Shirokoff et al. 2011), and in early data from Planck (Planck Collaboration et al. 2011b, hereafter P11). Correlations between clustering at different frequencies have also been detected: Hajian et al. (2012, hereafter H12) measure significant levels of correlation between BLAST maps and ACT maps at 148 and $218 \mathrm{GHz}$, 
detecting a clustered component at $4 \sigma$. P11 also find a significant correlation between the Planck $217 \mathrm{GHz}$ maps and those at higher frequencies $(353,545$, and $857 \mathrm{GHz})$. Studying the clustered power of dusty galaxies in the submillimeter is simpler than at the millimeter CMB bands because they are the dominant extragalactic signal. Knowing that significant overlap exists between the submillimeter galaxy population and those sources responsible for the IR foreground in the CMB bands suggests that information about the former can help our understanding of the latter.

In this work, we combine large- and small-scale power spectra from Planck, BLAST, and correlations between BLAST and ACT to estimate the amplitude and scale dependence of the angular power spectrum of clustered IR sources. We present a simple power-law template to model the clustered source contribution that may be marginalized over when estimating cosmological parameters from foreground-contaminated CMB maps as in, for instance, Hall et al. (2010), Dunkley et al. (2011), and Keisler et al. (2011).

Previous ACT and SPT results (Dunkley et al. 2011; Shirokoff et al. 2011) have found that the parameters extracted from their CMB spectra are not particularly dependent on the model adopted for the IR clustered power (see also Sehgal et al. 2010; Fowler et al. 2010). The ACT and SPT data sets are not yet complete; the final data will include more sky coverage as well as measurements from additional frequency channels. Millea et al. (2012) find that modeling the IR point-source clustering incorrectly for the final combined Planck and ACT/SPT data sets could introduce a significant bias in cosmological parameters (they estimate $1 \sigma$ based on the discrepancy between two different IR clustering models). It is therefore important to understand the scale and frequency dependence of the clustered power in preparation for this future analysis. Improving constraints on IR clustering will also help constrain the SZ power spectrum.

In this work, we are primarily concerned with the IR sources as a contaminant in CMB maps. While the physical properties of this high-redshift star-forming population are important for understanding the star formation history and galaxy evolution, in this analysis we do not attempt to extract information about, for example, the redshift distribution of the sources or the dark matter halos they occupy.

In Section 2 we describe the data we use for our fitting, in Section 3 we explain our assumptions and methods, results are presented in Section 4, and a conclusion follows in Section 5.

\section{DATA}

Throughout this work we use "auto-spectrum" to refer to a power spectrum calculated by correlating two maps at the same frequency, and "cross-spectrum" to refer to a spectrum calculated by cross-correlating maps at different frequencies. "BLAST $\times$ ACT" is to be understood to refer to the BLAST/ACT cross-spectra and so on.

We use the BLAST 250, 350, and $500 \mu \mathrm{m}$ auto-spectra, $250 \times$ $350,250 \times 500$, and $350 \times 500 \mu \mathrm{m}$ cross-spectra, and BLAST 250, 350, and $500 \mu \mathrm{m} \times$ ACT 148 and $218 \mathrm{GHz}$ cross-spectra from H12, and Planck 857, 545, 353, and $217 \mathrm{GHz}$ auto-spectra from P11 to construct the template. The ACT data are from the 2008 observing season and the BLAST $\times$ ACT spectra were calculated from the $\sim 8.6 \mathrm{deg}^{2}$ common to both sets of maps, as described in H12. We take the quadrature sum of the statistical and beam systematic uncertainties in Table 4 of P11 as the error on each Planck data point, neglecting any possible correlation in the beam uncertainty across different angular scales. We find that allowing such a correlation has minimal effect on our results (Section 4.1.3). The BLAST and ACT beam uncertainties are subdominant to the statistical (noise) uncertainty across the range of angular scales covered by the BLAST and BLAST $\times$ ACT data and so we likewise neglect any correlation in the errors on these spectra. The temperature-toflux conversion factors given in Table 4 of P11 assume a source spectral energy distribution (SED) that varies as $I(v) \propto v^{-1}$; a correction is then applied to convert to the real flux units (see Section 5.5 of P11 and Section 7.4.2 of Planck HFI Core Team et al. 2011).

We subtract the Galactic dust emission (cirrus) component in the BLAST and BLAST $\times$ ACT spectra as described in Section 4.3 of H12, assuming the cirrus contribution varies with angular scale as $\ell^{-2.7}$. These spectra are not very sensitive to the details of the cirrus treatment, since they are from a relatively cirrus-free patch of sky (see also Das et al. 2011). We assume that any contribution to the power spectra other than that from IR galaxies (for instance, radio-galaxy-IR or SZ-IR correlations) is negligible. Removal of power in the Planck spectra that is not from extragalactic IR point sources is described in Sections 2 and 3 of P11.

The Planck and BLAST auto-spectra are presented in Figure 1. We also show the $218 \mathrm{GHz}$ ACT power spectrum from Das et al. (2011) and the $220 \mathrm{GHz}$ SPT spectrum from Shirokoff et al. (2011). A WMAP-7 best-fit $\Lambda$ CDM CMB power spectrum (Komatsu et al. 2011) has been subtracted from these points; we have not subtracted any power from radio point sources or the kinetic SZ effect, as these contributions are likely to be subdominant (Dunkley et al. 2011; Shirokoff et al. 2011). All the spectra show similar angular scale dependence despite spanning a broad range of frequencies and almost two decades in angular scale. Note that a color correction to account for the different bandpass filters is required to make the Planck and BLAST spectra directly comparable-see Section 4 for details.

The Planck, BLAST, and ACT maps are calibrated using comparisons to various measurements including the orbital $\mathrm{CMB}$ dipole, FIRAS data, and planetary temperature. Uncertainty in these calibrations must be accounted for in order to perform joint fits to the power spectra extracted from the different maps. The absolute photometric calibration uncertainties are $7 \%$ for the Planck 857 and $545 \mathrm{GHz}$ maps, 2\% for the Planck 353 and $217 \mathrm{GHz}$ maps (see P11 and Planck HFI Core Team et al. 2011), $9.5 \%, 8.7 \%$, and $9.2 \%$ for the BLAST 250,350 , and $500 \mu \mathrm{m}$ maps (Truch et al. 2009), 7\% for the ACT $218 \mathrm{GHz}$ map, and $2 \%$ for the ACT $148 \mathrm{GHz}$ map (Hajian et al. 2011). Furthermore, the uncertainties in the BLAST calibrations are highly correlated; this is because the dominant source of uncertainty is the SED of the star used for the calibration of all three bands (Truch et al. 2009).

\section{POWER-LAW CLUSTERING TEMPLATE}

In the halo model formalism (e.g., Peacock \& Smith 2000; Scoccimarro et al. 2001; Cooray \& Sheth 2002; see also earlier work, e.g., Bond 1996, and references therein), the two-point function of galaxy clustering is dominated on large scales by pairs of sources in different dark matter halos (the "two-halo" term-roughly corresponding to the linear clustering regime), while on small scales galaxy pairs occupying the same halo ("one-halo" term-nonlinear regime) become dominant. Although these two components have different scale dependence, an angular correlation function varying as 


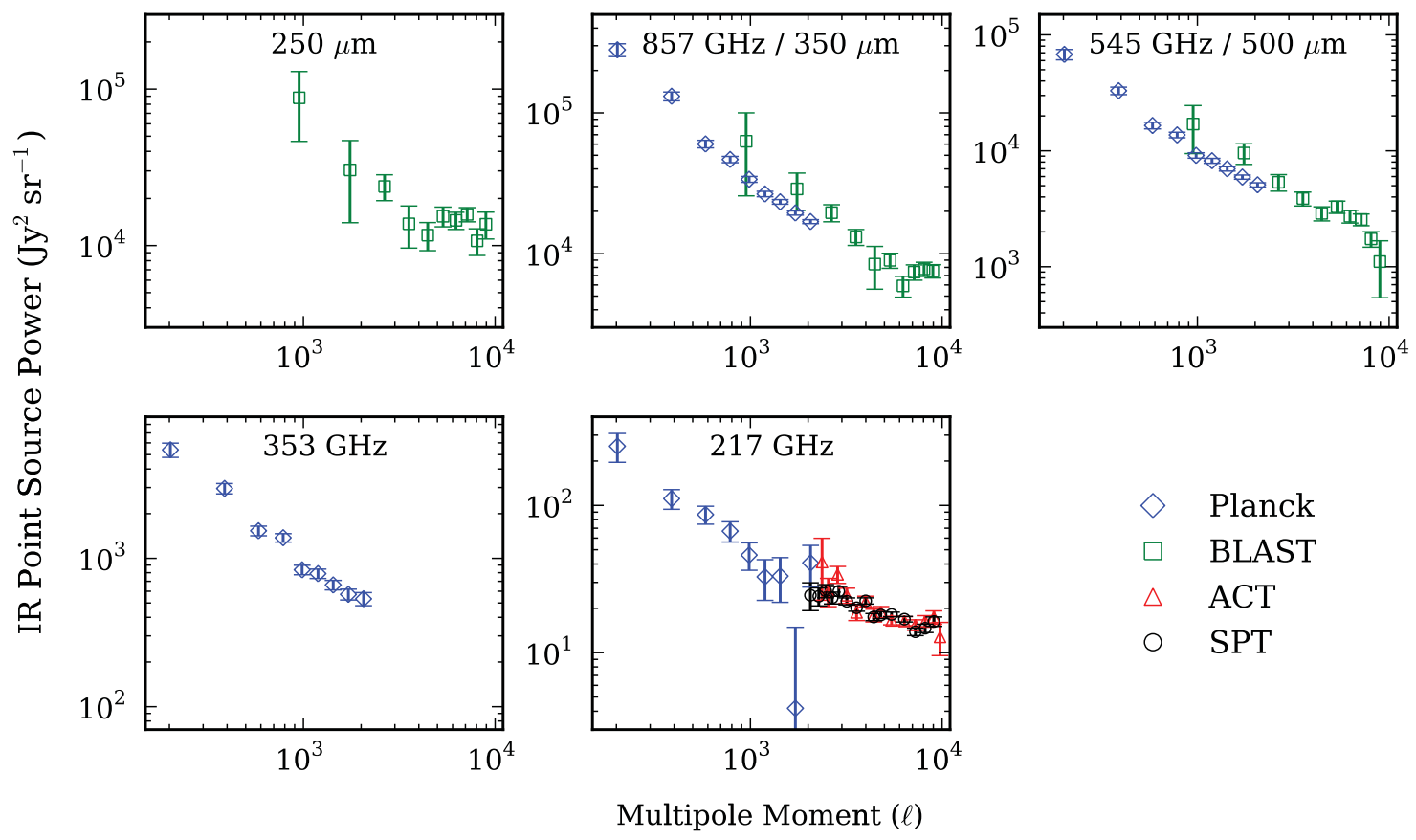

Figure 1. Planck $(857,545,353$, and $217 \mathrm{GHz})$ and BLAST $(250,350$, and $500 \mu \mathrm{m})$ IR point-source power spectra. The spectra include both shot noise and clustered components. While these data span a broad range of frequency and angular scale there is a notable similarity in the angular scale dependence. The Planck error bars include both the statistical uncertainties and estimates of the systematic beam uncertainty given in Table 4 of Planck Collaboration et al. (2011b). Data points at $\ell \geq 2000$ from ACT at $218 \mathrm{GHz}$ (Das et al. 2011) and SPT at $220 \mathrm{GHz}$ (Shirokoff et al. 2011) have been included for comparison with the Planck $217 \mathrm{GHz}$ spectrum. We have subtracted a WMAP-7 best-fit $\Lambda$ CDM CMB component from these spectra. No corrections for the different bandpass filter profiles have been applied; when the different filters and photometric calibration uncertainties are accounted for the Planck and BLAST data are in good agreement (Section 4).

(A color version of this figure is available in the online journal.)

$w(\theta) \propto \theta^{-\delta}$ with $\delta \sim 0.8$ (Peebles 1980, corresponding to clustering power $C_{\ell}^{\text {clust }} \propto \ell^{-1.2}$ ) has been found to adequately describe the clustering of, for instance, Lyman break galaxies at $z \sim 3$ (Giavalisco et al. 1998) and local SDSS galaxies (Zehavi et al. 2002). Significant deviations from power-law behavior have however been observed in analyses of more recent and comprehensive SDSS data (e.g., Zehavi et al. 2004, 2005; Blake et al. 2008), as well as in high-redshift galaxies (Ouchi et al. 2005; Lee et al. 2006; Coil et al. 2006; Wake et al. 2011). For a recent discussion of the physical origins of power-law galaxy correlation functions see Watson et al. (2011).

P11 and Amblard et al. (2011) find that a power law provides an adequate fit to the existing Planck and Herschel/SPIRE unresolved IR point-source spectra (see Tables 6 and S1 in those papers, respectively). This would suggest that IR spectra are not yet of sufficient quality to reveal deviations from power-law clustering behavior and we therefore also adopt a power law for the clustering component in this work.

We model the total IR point-source power spectrum from correlating maps at frequencies $v_{1}$ and $v_{2}\left(v_{1}=v_{2}\right.$ for the auto-spectra) as

$$
C_{\ell}\left(v_{1}, v_{2}\right)=A_{\mathrm{c}}\left(v_{1}, v_{2}\right)\left(\frac{\ell}{\ell_{0}}\right)^{-n}+C_{\mathrm{P}}\left(v_{1}, v_{2}\right),
$$

where $\ell$ is the multipole moment, $A_{\mathrm{c}}$ and $n$ are the clustering amplitude and index, $C_{\mathrm{P}}$ is the Poisson shot noise, and $\ell_{0}=$ 3000 is the pivot scale. This differs from the form adopted by Amblard et al. (2011) only in the choice of pivot $\ell_{0}$. Unlike P11 we model the clustering and shot noise as separate, independent components rather than modeling their sum as one power law. Motivated by the apparent uniformity in angular scale dependence across the different spectra (see Figure 1), we fit for a single, frequency-independent, value of $n$. We fit for a separate shot-noise level in each of the 16 Planck, BLAST, and BLAST $\times$ ACT spectra (see Section 3.2).

The SED of the CIB, over the range of frequencies considered, has been found to be well described by a modified blackbody of the form (e.g., Fixsen et al. 1998; Lagache et al. 1999; Gispert et al. 2000)

$$
I_{\mathrm{CIB}}(v) \propto v^{\beta} B\left(v, T_{\mathrm{eff}}\right) .
$$

In reality, the sources making up the CIB lie at a range of redshifts, with a variety of luminosities and dust temperatures (e.g., Haiman \& Knox 2000; Knox et al. 2001; Coppin et al. 2008; Pascale et al. 2009; Hwang et al. 2010). Equation (2) is thus an approximation to the true CIB SED, which consists of the sum of many different (approximately) modified blackbody spectra, and the quantities $\beta$ and $T_{\text {eff }}$ are not to be interpreted as physical parameters.

P11 found that the SED of the CIB anisotropies measured by Planck is also well described by the Gispert et al. (2000) modified blackbody with emissivity spectral index $\beta=1.4 \pm$ 0.2 and effective temperature $T_{\text {eff }}=13.6 \pm 1.5 \mathrm{~K}$. We assume that the clustering power SED can also be described in this form and parameterize the frequency dependence of the autospectrum clustering power amplitude as $A_{\mathrm{c}}=\left(I_{\mathrm{c}}\right)^{2}$ where

$$
I_{\mathrm{c}}(\nu)=I_{0}\left(\frac{v}{v_{0}}\right)^{\beta} B\left(\nu, T_{\mathrm{eff}}\right),
$$

with a single emissivity index and effective temperature.

Different frequencies are sensitive to IR sources at different redshifts, with the importance of higher-redshift sources increasing at lower frequencies (e.g., Haiman \& Knox 2000; Knox et al. 2001; Chapin et al. 2009; Marsden et al. 2009). As a result we 
would not expect there to be $100 \%$ correlation between the IR sources in different bands (particularly between the widely spaced ACT and BLAST bands) and we therefore include a clustering correlation factor $f_{\text {corr }}$ as a free parameter for each cross-spectrum. The cross-spectrum clustering amplitude is then $A_{\mathrm{c}}\left(v_{1}, v_{2}\right)=f_{\text {corr }}\left(v_{1}, v_{2}\right) I_{\mathrm{c}}\left(v_{1}\right) I_{\mathrm{c}}\left(v_{2}\right)$.

\subsection{Shot Noise}

The auto-spectrum shot noise is given by (e.g., Scott \& White 1999)

$$
C_{\mathrm{P}}=\int d z \int_{0}^{S_{\mathrm{cut}}} d S S^{2} \frac{d^{2} N}{d S d z}(S, z),
$$

where $S_{\text {cut }}$ is the flux cut applied to the map and $d^{2} N / d S d z$ are the differential source counts.

In P11 the shot-noise levels were fixed using the IR galaxy evolution model of Béthermin et al. (2011, hereafter B11). This model parameterizes the evolution of the galaxy luminosity function and is fitted to number counts over a wide range of IR wavelengths. While the model broadly fits the available data, there are discrepancies. The model underpredicts by $\sim 40 \%$ the shot-noise levels measured at $500 \mu \mathrm{m}$ by BLAST (Viero et al. 2009) and $220 \mathrm{GHz}$ by SPT (Hall et al. 2010). We do not apply any priors on the shot-noise levels when constructing our template. We may, however, expect a considerable degeneracy between the shape of the clustering component and size of the shot noise for the Planck data, because Planck is not able to probe the small scales where the shot noise becomes dominant. As a result, we consider the effect of adopting the B11 model predictions as priors on the Planck shot-noise levels in Section 4.1.1.

In terms of the source flux and $S_{\text {cut }}$ we can write the clustering power as (e.g., Tegmark et al. 2002; Viero et al. 2009)

$$
C_{\ell}^{\text {clust }}=\left.\int d z\left(\frac{d V}{d z}(z)\right)^{-1}\left(\frac{d S}{d z}(z)\right)^{2} P_{\text {gal }}(k, z)\right|_{k=\ell / \chi(z)},
$$

where $\chi$ is the comoving distance, $d V / d z=\chi^{2} d \chi / d z$ is the comoving volume element, $P_{\text {gal }}$ is the IR galaxy power spectrum, and the redshift distribution of the flux, $d S / d z$, is given by

$$
\frac{d S}{d z}(z)=\int_{0}^{S_{\text {cut }}} d S S \frac{d^{2} N}{d S d z}(S, z)
$$

The factor of $S^{2}$ in Equation (4), compared to $(d S / d z)^{2}$ in Equation (5), suggests that the removal of the highest-flux sources will have much less impact on the clustered power than on the shot noise. The B11 model predicts that, for instance, applying a flux cut of $250 \mathrm{mJy}$ (the cut applied to the BLAST $350 \mu \mathrm{m}$ map in H12) to the Planck $857 \mathrm{GHz}$ map would result in a $\sim 10 \%$ reduction in the shot-noise level compared to the Planck flux cut of $710 \mathrm{mJy}$, but that $(d S / d z)^{2}$ would be reduced by $<1 \%$ at $z \sim 0.2$ and virtually unaffected for $z>1$. We allow for the dependence of the shot-noise levels on flux cut by fitting separate shot-noise levels for each spectrum, as described in the next section. We assume that the effect on the clustering power from applying different flux cuts is negligible for the data considered. Studies using higher-resolution Herschel maps will be able to investigate the dependence of the clustering power on flux cut in more detail.

\subsection{MCMC Fitting}

We perform a simultaneous fit to the seven auto-spectra (four Planck and three BLAST) plus the nine cross-spectra (three
BLAST $\times$ BLAST and six BLAST $\times$ ACT). The model spectra are binned for comparison to each data spectrum, with likelihood

$$
-2 \ln L(d \mid \theta)=\sum_{i=1}^{16}\left[\mathbf{C}_{i}^{\text {data }}-\mathbf{C}_{i}^{\text {model }}(\theta)\right]^{2} / \boldsymbol{\sigma}_{i}^{2},
$$

for model parameters $\theta$, data vector $\mathbf{C}_{i}^{\text {data }}$ for the $i$ th spectrum, and binned model spectra $\mathbf{C}_{i}^{\text {model }}(\theta)$. Covariances between the 16 spectra are neglected.

We find that the clustering SED parameters $I_{0}, \beta$, and $T_{\text {eff }}$ (Equation (3)) are highly degenerate. Only two of these parameters are really independent and so we fix $T_{\text {eff }}$ to the bestfit value, $9.7 \mathrm{~K}$. We choose $v_{0}=530 \mathrm{GHz}$ to minimize the degeneracy between $I_{0}$ and $\beta$.

We therefore fit for 37 parameters: $n$, the clustering index, $I_{0}$, $\beta, 16$ shot-noise levels (one for each spectrum), nine crossspectrum correlation factors (one for each cross-spectrum), and nine photometric calibration parameters (one for each Planck, BLAST, and ACT band). For each calibration factor we enforce a Gaussian prior centered at unity, with spread given by the nominal uncertainty listed in Section 2. The covariance between the BLAST calibration factors from Truch et al. (2009) is included. Apart from the calibration factors, all priors are uniform.

Given the high dimensionality, we estimate the posterior probability distribution using Markov Chain Monte Carlo (MCMC) analysis (Metropolis et al. 1953), using the sampling methods and convergence test described in Dunkley et al. (2005). Chains used for analysis are about $10^{6}$ steps in length, and are used to calculate one-dimensional marginalized parameter values and errors.

To judge the goodness of fit of the model, we are also interested in the maximum likelihood. However, the peak of the likelihood distribution occupies only a small part of this highdimensional parameter space, so the minimum $\chi^{2}$ sampled in a chain of about a million steps is significantly larger than the true value $\left(\Delta \chi^{2} \sim 10\right.$ for a simulated 37-dimensional Gaussian distribution). There are numerous statistical methods to find the true peak of a distribution. We adopt a simple modification to the Metropolis algorithm in which chains start from the best-fitting point and then make a step in parameter space only when the posterior is improved, using a reduced trial step size. We have tested this prescription on simulated Gaussian distributions, and the peak is found to within $\Delta \chi^{2}=0.1$ in $\sim 2 \times 10^{4}$ steps for 37 dimensions. We emphasize that this modified code is used only to assess the global goodness of fit, not to estimate the marginalized parameter distributions.

To estimate the model spectra at each frequency, it is not sufficient to use the nominal values of $v$ for the BLAST and ACT bands in Equation (3), due to the finite width of each filter. The correct effective $v$ values depend on the SED of the emission mechanism. The clustering SED is estimated iteratively by repeating the MCMC fitting; each time the best-fit clustering SED is integrated through the BLAST and ACT filter profiles and an improved estimate of the effective $v$ values obtained. We found that after three iterations the SED converged, with effective frequencies of 1248, 829, and $607 \mathrm{GHz}$ (effective wavelengths 240, 362, and $494 \mu \mathrm{m}$ ) for the nominal BLAST 250, 350, and $500 \mu \mathrm{m}$ bands, and 220 and $150 \mathrm{GHz}$ for the ACT 218 and $148 \mathrm{GHz}$ bands. While the clustering SED is rising steeply at the ACT bands, the shift from nominal frequency is reduced by the narrowness of the ACT filters. For the Planck data the nominal frequencies are used, because the temperature- 
Table 1

Power-law Clustering Template

\begin{tabular}{lc}
\hline \hline Parameter & Value \\
\hline$n^{\mathrm{a}}$ & $1.25 \pm 0.06$ \\
$I_{0}\left(\mathrm{Jy} \mathrm{sr}^{-1}\right)^{\mathrm{a}}$ & $(2.43 \pm 0.16) \times 10^{-9}$ \\
$\beta$ & $2.20 \pm 0.07$ \\
$T_{\text {eff }}(\mathrm{K})$ & 9.7 \\
$\nu_{0}(\mathrm{GHz})$ & 530 \\
$\ell_{0}$ & 3000 \\
\hline$\chi^{2} /$ dof & $132 / 122$ \\
\hline
\end{tabular}

Note. ${ }^{a}$ A strong (89\%) anti-correlation exists between $n$ and $I_{0}$.

to-flux conversion factors provided in P11 are given such that the flux is correct at the nominal frequencies.

\section{RESULTS}

The model fits the data well. We find a best-fit $\chi^{2}$ of 132 for 122 degrees of freedom (150 data points minus 28 parameters; we have not counted the calibration parameters since they are strongly constrained by priors), giving reduced- $\chi^{2}=1.08$. The marginalized mean values of the clustered IR template parameters are given in Table 1 . No errors are given for $T_{\text {eff }}, v_{0}$, or $\ell_{0}$ since these parameters are fixed as described in Section 3.2. Figure 2 shows the best-fit clustered IR source power for each of the bands included in the fit. The best-fit shot-noise levels have been subtracted. The $\chi^{2}$ contribution from each individual spectrum is also shown; in addition to the total $\chi^{2}$ being good we find that there are no spectra which are not individually well fitted by the model.

We plot the BLAST 350 and $500 \mu \mathrm{m}$ spectra on the same axes as the Planck 857 and $545 \mathrm{GHz}$ spectra. The BLAST spectra are color-corrected to account for the difference in the bandpass filters by taking the ratio of our clustering power predictions for the relevant Planck and BLAST bands. We find these color correction factors to be 1.07 and 0.63 for the 350 and $500 \mu \mathrm{m}$ BLAST spectra, respectively. P11 found values of 1.05 and 0.7 by integrating the SED of Gispert et al. (2000) through the BLAST and Planck filters. These values differ slightly because our best-fit clustering SED is slightly different-see Figure 3 for a comparison.

The clustering power at $\ell=3000$ is shown as a function of frequency in Figure 3. Also shown is the scaled Gispert et al. (2000) SED (dashed line), which was fitted to FIRAS and DIRBE measurements of the CIB intensity spectrum. It falls off more slowly with decreasing frequency than our clustering SED. Some difference between the CIB mean and anisotropy SED may be expected, since the contribution of a source to the anisotropy SED depends on its clustering as well as spectral properties.

IR clustering power predictions at $\ell=2000$ and 3000, calculated from our template for several Planck, ACT, and SPT bands, are given in Table 2. The conversion from flux to temperature units was calculated in each case by integrating $I_{\mathrm{c}}$ from Equation (3) through the relevant bandpass filter. The effective frequencies (i.e., single frequency value that gives the same clustering amplitude as integrating through the filter) are also given. Since the IR clustering amplitude is rising strongly with frequency at the CMB bands (see Figure 3), the different filter profiles lead to significant differences in the clustering power even for bands with closely spaced nominal frequencies.
Table 2

IR Clustering Predictions from Our Template $-\ell(\ell+1) C_{\ell}^{\text {clust }} / 2 \pi\left(\mu \mathrm{K}^{2}\right)$

\begin{tabular}{lccc}
\hline \hline Band & $\begin{array}{c}v_{\text {eff }} \\
(\mathrm{GHz})\end{array}$ & $\ell=2000$ & $\ell=3000$ \\
\hline Planck $100 \mathrm{GHz}$ & 104 & $0.49 \pm 0.07$ & $0.67 \pm 0.09$ \\
Planck $143 \mathrm{GHz}$ & 146 & $2.9 \pm 0.3$ & $3.9 \pm 0.4$ \\
Planck $217 \mathrm{GHz}$ & 226 & $45 \pm 4$ & $61 \pm 6$ \\
ACT $148 \mathrm{GHz}$ & 150 & $3.2 \pm 0.4$ & $4.4 \pm 0.5$ \\
ACT $218 \mathrm{GHz}$ & 220 & $36 \pm 3$ & $49 \pm 4$ \\
SPT $95 \mathrm{GHz}$ & 99 & $0.38 \pm 0.05$ & $0.51 \pm 0.07$ \\
SPT $150 \mathrm{GHz}$ & 156 & $4.1 \pm 0.4$ & $5.5 \pm 0.6$ \\
SPT $220 \mathrm{GHz}$ & 221 & $37 \pm 3$ & $50 \pm 5$ \\
\hline
\end{tabular}

The amplitude of the clustered power may vary by a factor of ten across a single filter, as shown in Figure 3.

The predictions at $\ell=3000$ can be compared to the ACT results from Dunkley et al. (2011) and SPT results from Shirokoff et al. (2011). Dunkley et al. find a best-fit clustering amplitude of $4.6 \pm 1.1$ and $54 \pm 13 \mu \mathrm{K}^{2}$ at 148 and $218 \mathrm{GHz}$, respectively (we have calculated these uncertainties by adding their statistical and systematic error estimates in quadrature). Shirokoff et al. find $6.1 \pm 0.8$ and $57 \pm 8 \mu \mathrm{K}^{2}$ at 150 and $220 \mathrm{GHz}$, respectively, for their base-line model, which includes a power-law IR clustering component with index $n=1.2$. Our predictions are in excellent agreement with these results. Figure 4 shows ACT and SPT data at $220 \mathrm{GHz}$ with our clustering template predictions overplotted, along with an IR shot-noise component and a $\Lambda$ CDM CMB power spectrum.

We also make predictions for the spectral index $\alpha$ of the clustered component. At the CMB frequencies the clustering SED can be approximated as a power law, $I_{\mathrm{c}}(v) \propto v^{\alpha}$; however, the CMB bands do not lie strictly in the Rayleigh-Jeans limit of the modified blackbody adopted in our model. Consequently the equivalent power-law slope at $150 \mathrm{GHz}$ differs from that at $220 \mathrm{GHz}$ : we find $\alpha_{150}=3.78 \pm 0.07$ and $\alpha_{220}=3.56 \pm 0.07$. We also calculate an effective spectral index between 150 and $220 \mathrm{GHz}, \alpha_{150-220}=3.68 \pm 0.07$. This result is consistent with ACT and SPT findings of $3.69 \pm 0.14$ and $3.58 \pm 0.08$, respectively.

We have considered only the frequency dependence of the IR clustering component, not the shot noise. Investigating shotnoise frequency dependence is more difficult, due to stronger flux cut dependence and the fact that the Planck data are limited to large scales; future Herschel, Planck, ACT, SPT, and crosscorrelation data will help overcome these issues.

\subsection{Validating Assumptions}

While our single-index power-law clustering model provides a good fit to the data in terms of $\chi^{2}$, in this section we attempt to further validate our assumptions regarding the scale and frequency dependence of the clustered power.

\subsubsection{Shot Noise}

Table 3 shows the one-dimensional marginalized values of the shot-noise levels from our MCMC chains $\left(C_{\mathrm{P}}\right.$ from Equation (1)). It should be noted that, as expected, the Planck shot-noise levels and the clustering index $n$ are highly correlated. The shot-noise predictions for each auto-spectrum from the B11 model are given for comparison. Our values are consistent with the model within $1.5 \sigma$ in all cases. We repeated the MCMC analysis described in Section 3.2 using the B11 model 
Auto-Spectra
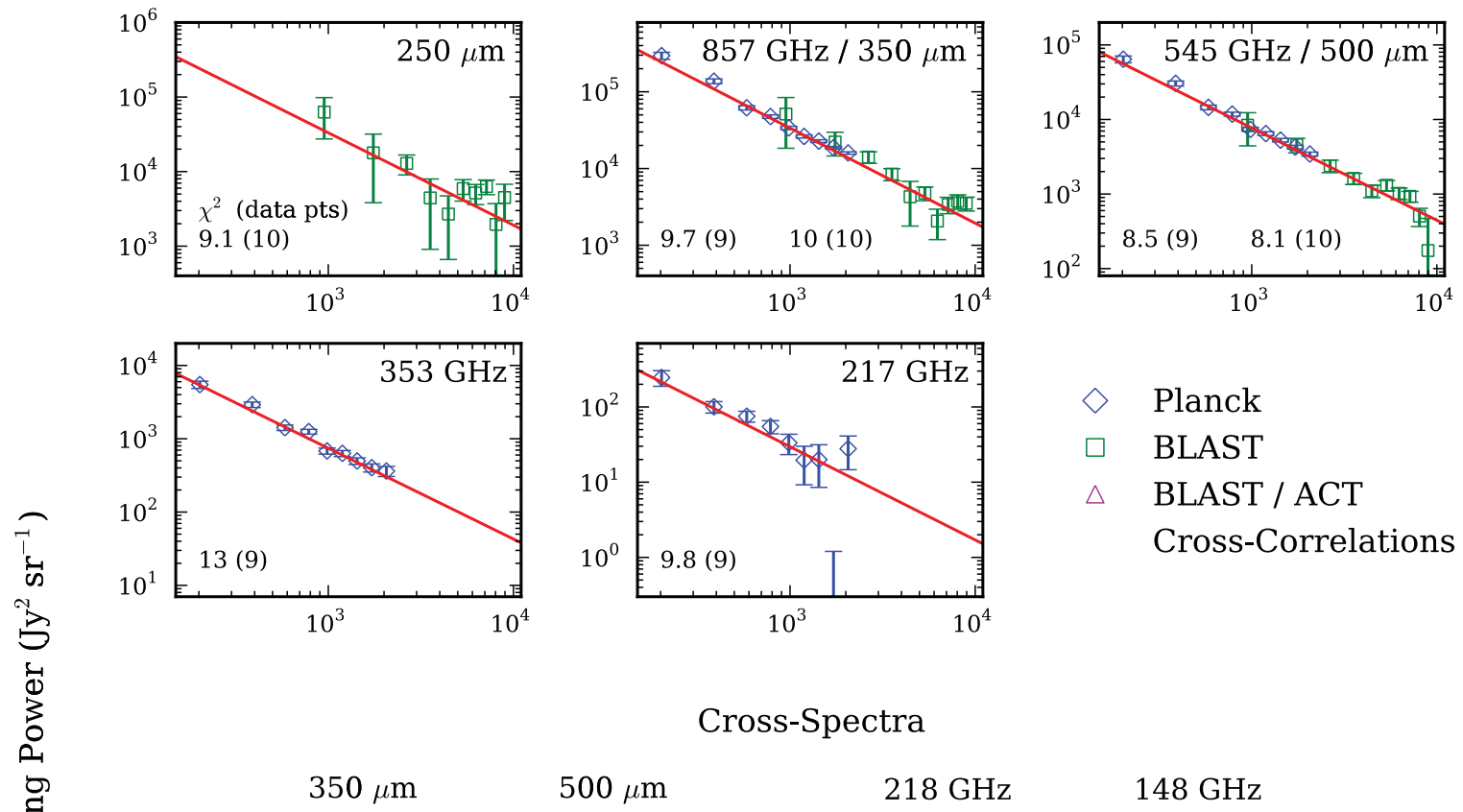

Cross-Spectra
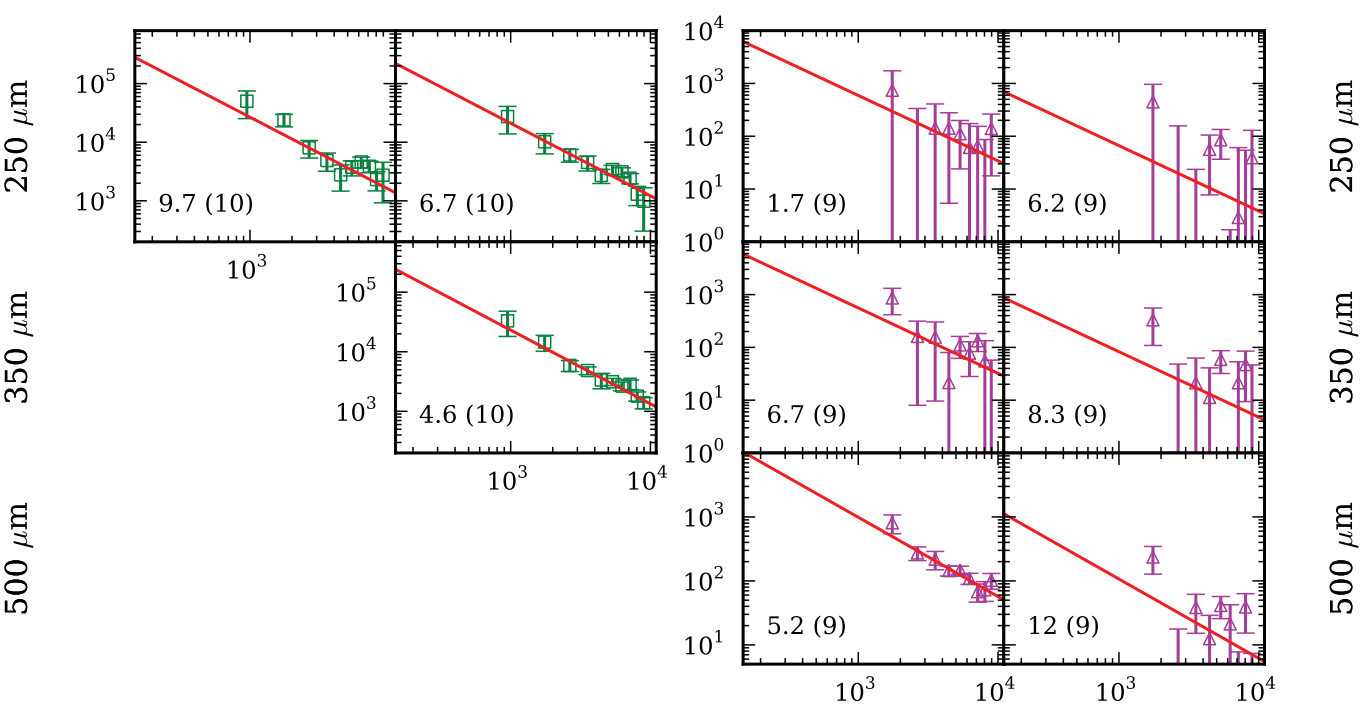

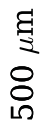

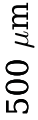

Multipole Moment $(\ell)$

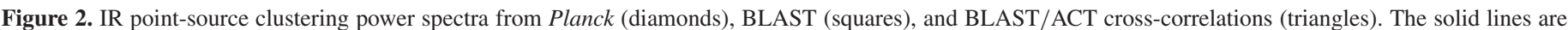

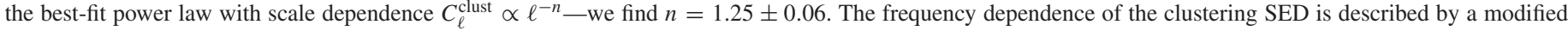

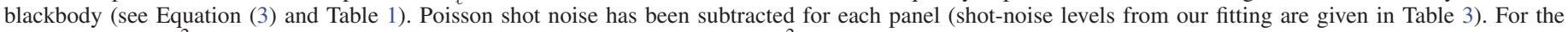

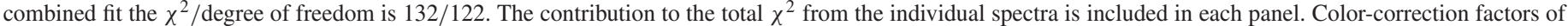

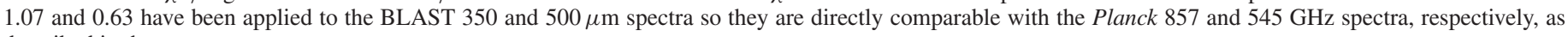
described in the text.

(A color version of this figure is available in the online journal.)

predictions as Gaussian priors on the Planck shot-noise levels and found a best-fit $\chi^{2} /$ dof $=139 / 122$, an increase of $\Delta \chi^{2}=7$ compared to the case with uniform priors. There was minimal change in the clustering template parameters (e.g., $n=1.24 \pm$ 0.03 with the shot-noise prior). This suggests that uncertainty in the Planck shot-noise levels is not having a significant impact on our results.

\subsubsection{Photometric Calibration}

The marginalized values for the photometric calibration parameters from our MCMC chains are shown in Table 4 along with the nominal uncertainties. We would expect the calibration parameters to be consistent with unity within the nominal uncertainty. If this were not the case it could indicate that the modified blackbody spectrum in Equation (3) was not a suitable description for the frequency dependence of the clustering amplitude; however all are consistent with the nominal values.

\subsubsection{Planck Beam Uncertainty}

As stated in Section 2, we neglected any possible correlation in the Planck beam uncertainty across different angular scales. 


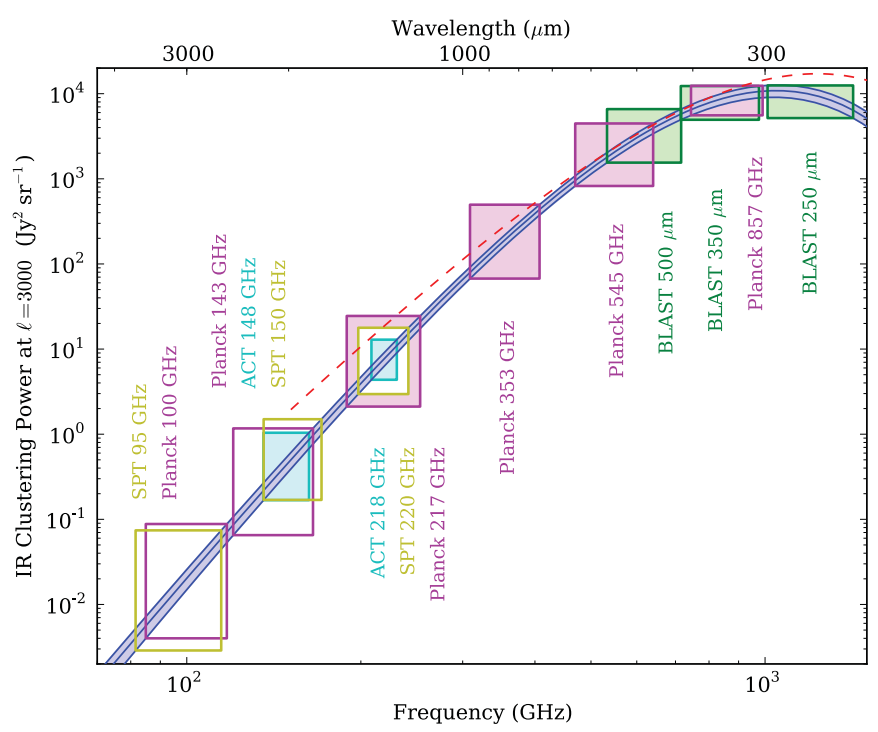

Figure 3. Frequency dependence of IR point-source clustering power at $\ell=3000$. We assume this frequency dependence can be described by a modified blackbody: $C_{\ell=3000}^{\text {clust }}(v) \propto\left[v^{\beta} B\left(\nu, T_{\text {eff }}\right)\right]^{2}$. The solid lines show the best-fit and $1 \sigma$ uncertainties from our fitting; parameter values are given in Table 1. The dashed line is the SED of Gispert et al. (2000), which has been scaled for comparison. The rectangles show the Planck, BLAST, ACT, and SPT bandpass filter FWHM values in the horizontal direction. The vertical extent of the rectangles shows the variation of the clustering power across each filter; in some cases, this variation is a factor of ten or more due to how steeply the clustering SED rises with frequency. The shaded rectangles show the bands that were used for the fitting in this paper. Spectra from the other bands are either not yet available or were not used, due to the risk of biasing results by assumptions regarding the separation of the $\mathrm{CMB}$ and other components from the IR contribution.

(A color version of this figure is available in the online journal.)

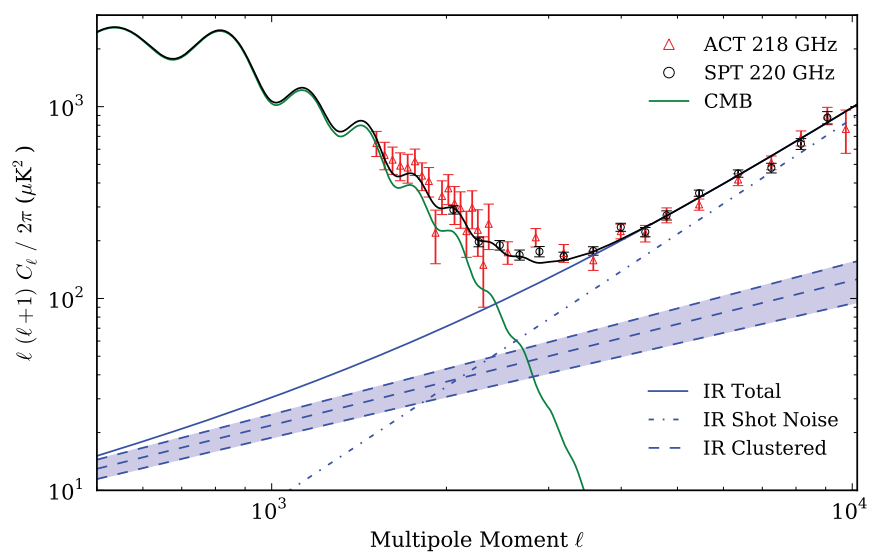

Figure 4. Angular power spectra at $220 \mathrm{GHz}$ measured by ACT (Das et al. 2011) and SPT (Shirokoff et al. 2011), with a theoretical model for CMB and IR point sources overplotted. The clustered IR component is given by the powerlaw model fit to Planck, BLAST, and BLAST $\times$ ACT data in this analysis (see Equations (1) and (3) and Table 1 for parameter values). The upper and lower dashed lines correspond to $1 \sigma$ error bounds. The lensed CMB spectrum is that of the $\Lambda C D M$ model with parameters derived from WMAP (Komatsu et al. 2011). An IR shot-noise component of size $\ell(\ell+1) C_{\mathrm{P}} /\left.2 \pi\right|_{\ell=3000}=78 \mu \mathrm{K}^{2}$ (consistent with ACT and SPT measurements) has also been plotted. Additional radio source and kinetic SZ power are subdominant at this frequency and are not included.

(A color version of this figure is available in the online journal.)

We re-ran the MCMC chain enforcing a $100 \%$ correlation in the beam uncertainty in different $\ell$-bins for each Planck spectrum (introducing off-diagonal elements in the likelihoodEquation (7)) and found minimal change in the marginalized parameter values ( $<0.3 \sigma$ in all cases). While the Planck beam
Table 3

One-dimensional Marginalized Shot-noise Levels

\begin{tabular}{lcc}
\hline \hline Band & $\begin{array}{c}\text { Marginalized Value } \\
\left(\mathrm{Jy}^{2} \mathrm{sr}^{-1}\right)\end{array}$ & $\begin{array}{c}\text { Béthermin et al. (2011) } \\
\left(\mathrm{Jy}^{2} \mathrm{sr}^{-1}\right)\end{array}$ \\
\hline Planck $857 \mathrm{GHz}$ & $2200 \pm 2500$ & $5920 \pm 370$ \\
Planck $545 \mathrm{GHz}$ & $1700 \pm 700$ & $1150 \pm 90$ \\
Planck $353 \mathrm{GHz}$ & $210 \pm 60$ & $138 \pm 22$ \\
Planck $217 \mathrm{GHz}$ & $16 \pm 6$ & $12 \pm 3$ \\
BLAST $250 \mu \mathrm{m}$ & $11600 \pm 2300$ & \\
BLAST $350 \mu \mathrm{m}$ & $5200 \pm 1200$ & \\
BLAST $500 \mu \mathrm{m}$ & $1300 \pm 390$ & $1600 \pm 2100 \pm 1080$ \\
$250 \mu \mathrm{m} \times 350 \mu \mathrm{m}$ & $5700 \pm 1400$ & \\
$250 \mu \mathrm{m} \times 500 \mu \mathrm{m}$ & $1830 \pm 690$ & \\
$350 \mu \mathrm{m} \times 500 \mu \mathrm{m}$ & $820 \pm 490$ & \\
$250 \mu \mathrm{m} \times 218 \mathrm{GHz}$ & $240 \pm 130$ & \\
$250 \mu \mathrm{m} \times 148 \mathrm{GHz}$ & $90 \pm 70$ & \\
$350 \mu \mathrm{m} \times 218 \mathrm{GHz}$ & $240 \pm 70$ & \\
$350 \mu \mathrm{m} \times 148 \mathrm{GHz}$ & $110 \pm 40$ & \\
$500 \mu \mathrm{m} \times 218 \mathrm{GHz}$ & $66 \pm 30$ & \\
$500 \mu \mathrm{m} \times 148 \mathrm{GHz}$ & $55 \pm 20$ & \\
\hline
\end{tabular}

Table 4

One-dimensional Marginalized Calibration Parameters

\begin{tabular}{lcc}
\hline \hline Band & Marginalized Value & Nominal Uncertainty \\
\hline Planck $857 \mathrm{GHz}$ & $1.05 \pm 0.06$ & $7 \%$ \\
Planck $545 \mathrm{GHz}$ & $0.99 \pm 0.04$ & $7 \%$ \\
Planck $353 \mathrm{GHz}$ & $1.01 \pm 0.02$ & $2 \%$ \\
Planck $217 \mathrm{GHz}$ & $1.00 \pm 0.02$ & $2 \%$ \\
BLAST $250 \mu \mathrm{m}$ & $1.05 \pm 0.08$ & $9.5 \%$ \\
BLAST $350 \mu \mathrm{m}$ & $1.03 \pm 0.07$ & $8.7 \%$ \\
BLAST $500 \mu \mathrm{m}$ & $1.02 \pm 0.07$ & $9.2 \%$ \\
ACT 218 GHz & $1.03 \pm 0.07$ & $7 \%$ \\
ACT $148 \mathrm{GHz}$ & $1.00 \pm 0.02$ & $2 \%$ \\
\hline
\end{tabular}

uncertainties at $\ell \sim 2000$ are comparable to or larger than the statistical uncertainties, the template parameters are primarily constrained by the large-scale Planck data (where the beam uncertainties are sub-dominant) and the BLAST data.

\subsubsection{Comparison with Broken Power Law}

We assumed that the scale dependence of the clustering power can be described using a single index $n$. In this section, we consider fitting the scale dependence with a broken power law of the form

$$
C_{\ell}^{\text {clust }} \propto \begin{cases}\ell^{-n_{1}} & \text { if } \ell \leqslant \ell_{\mathrm{b}} \\ \ell^{-n_{2}} & \text { if } \ell>\ell_{\mathrm{b}},\end{cases}
$$

where $n_{1}, n_{2}$, and $\ell_{\mathrm{b}}$ are free parameters. When we repeat the fit to the Planck, BLAST, and BLAST $\times$ ACT spectra with this new scale dependence we find $n_{1}=1.24 \pm 0.06, n_{2}=1.2 \pm 0.2$, and $\ell_{\mathrm{b}}=1100 \pm 300$ with a best $\chi^{2} / \mathrm{dof}=131 / 120$. This is an improvement of only $\Delta \chi^{2}=0.9$ with two additional fitted parameters. For the fit with priors on the Planck shot-noise levels from the B11 model the improvement is only $\Delta \chi^{2}=0.4$. We conclude that the data do not show a significant preference for a broken power law.

Keisler et al. (2011) adopted a template of the form given in Equation (8) with $n_{1}=2.0, n_{2}=1.2$, and $\ell_{\mathrm{b}}=1500$ to model the IR clustered power when extracting cosmological information from the small-scale SPT $150 \mathrm{GHz}$ CMB power spectrum. We find that this form is not a good fit to the Planck data, with $\chi^{2} /$ dof $=22 / 7$ when we fit to the Planck $217 \mathrm{GHz}$ spectrum with $n_{1}, n_{2}$, and $\ell_{\mathrm{b}}$ fixed to the above values and using uniform priors on the clustering amplitude and shot noise. 
Table 5

Degree of Clustering Cross-correlation, $f_{\text {corr }}$

\begin{tabular}{lccc}
\hline \hline Band 1 & Band 2 & $\begin{array}{c}\Delta v \\
(\mathrm{GHz})\end{array}$ & Marginalized Value \\
\hline $250 \mu \mathrm{m}$ & $350 \mu \mathrm{m}$ & 340 & $0.98 \pm 0.30$ \\
$250 \mu \mathrm{m}$ & $500 \mu \mathrm{m}$ & 600 & $1.23 \pm 0.31$ \\
$350 \mu \mathrm{m}$ & $500 \mu \mathrm{m}$ & 260 & $1.41 \pm 0.28$ \\
$250 \mu \mathrm{m}$ & $218 \mathrm{GHz}$ & 982 & $0.66 \pm 1.09$ \\
$250 \mu \mathrm{m}$ & $148 \mathrm{GHz}$ & 1052 & $0.30 \pm 1.83$ \\
$350 \mu \mathrm{m}$ & $218 \mathrm{GHz}$ & 642 & $0.64 \pm 0.56$ \\
$350 \mu \mathrm{m}$ & $148 \mathrm{GHz}$ & 712 & $0.39 \pm 1.05$ \\
$500 \mu \mathrm{m}$ & $218 \mathrm{GHz}$ & 382 & $1.82 \pm 0.47$ \\
$500 \mu \mathrm{m}$ & $148 \mathrm{GHz}$ & 452 & $0.79 \pm 0.87$ \\
\hline
\end{tabular}

\subsubsection{Comparison with Linear Bias Model}

$\mathrm{H} 12$ found that the BLAST and BLAST $\times$ ACT data are well fitted by assuming the IR galaxy power spectrum in Equation (5) is given by

$$
P_{\text {gal }}(k, z)=b^{2} P_{\mathrm{DM}}(k, z),
$$

where $b$ is the linear bias factor and $P_{\mathrm{DM}}$ is the linear dark matter power spectrum, and using the B11 predictions for the redshift distribution of the flux, $d S / d z$. P11 also found that the Planck data from each band are well fitted by this model (also using the B11 $d S / d z$ ) if no prior is enforced on the shot-noise levels. The bias levels are not consistent; when fitting for a single, redshiftindependent value of $b, \mathrm{H} 12$ found $b=5.0 \pm 0.4$ whereas P11 found $b=2.18 \pm 0.11$ for the Planck $545 \mathrm{GHz}$ spectrum.

The linear bias model is strongly rejected by the combined Planck and BLAST data even without any shot-noise prior. Fitting a linear bias model with single-value bias to the Planck $857 \mathrm{GHz}$ and BLAST $350 \mu \mathrm{m}$ spectra together, using the B11 $d S / d z$ and multiplying the BLAST data points by a colorcorrection factor of 1.07 (see Section 4), yields $\chi^{2} /$ dof $=39 / 16$. For a joint fit to the Planck $545 \mathrm{GHz}$ and BLAST $500 \mu \mathrm{m}$ spectra we find $\chi^{2} /$ dof $=43 / 16$. This result is driven by the shape of the linear matter power spectrum rather than the choice of $d S / d z$ : we repeated the fitting using the predictions of Marsden et al. (2011) rather than B11 and found $\chi^{2} /$ dof of $36 / 16$ and $42 / 16$ for the $857 \mathrm{GHz} / 350 \mu \mathrm{m}$ and $545 \mathrm{GHz} / 500 \mu \mathrm{m}$ spectra, respectively. Neither the Planck nor BLAST data alone were able to rule out the linear bias model without a shot-noise prior because of the limited angular scales probed. Shirokoff et al. (2011) similarly found that the linear bias model could not be ruled out using only SPT data from $\ell \gtrsim 2000$.

\subsection{Cross-correlations}

Table 5 shows the marginalized degree of clustering correlation, $f_{\text {corr }}$, for each cross-spectrum included in our fitting, along with the separation $\Delta v$ between the two bands. The BLAST $\times$ BLAST cross-spectra are consistent with $100 \%$ correlation. Our conclusions regarding the degrees of correlation between the ACT and BLAST bands are limited by the data quality. A decrease in correlation with increasing band separation would be consistent with the sources lying at a range of redshifts, with the higher-redshift sources being of greater relative importance at the longer wavelengths (e.g., Haiman \& Knox 2000); however the current data are not of sufficient quality to confirm this.

Two of the marginalized mean $f_{\text {corr }}$ values lie more than $1 \sigma$ above unity, while none lie more than $1 \sigma$ below. Measuring $f_{\text {corr }}>1$ may indicate that the angular scale dependence of the cross-spectra clustering power is not described by the same single-index power law as the auto-spectra clustering, since there is no physical explanation for a correlation in excess of $100 \%$. This could be the case even with no worsening of the $\chi^{2}$, due to the limited angular scales probed by the BLAST $\times$ ACT data. To test that this is not having a significant effect on our clustering template, we repeated the MCMC fitting described in Section 3.2 using only the auto-spectrum data. We found that the values of $n, \beta$, and $I_{0}$ change by $<0.5 \sigma$ compared to the fit with the cross-spectra included. We conclude that while the data may be hinting that the cross-spectrum clustering power has a different shape to the auto-spectrum clustering, this is not significantly biasing our template.

Further submillimeter-millimeter cross-correlation studies (e.g., Herschel $\times$ ACT/SPT) are clearly required to provide more insight into the distribution of the sources with redshift, to constrain the angular scale dependence of the cross-spectrum clustering, and to investigate how the clustering shape changes with increasing band separation.

\section{CONCLUSIONS}

We have found that a power-law model for the IR point-source clustering is adequate to simultaneously fit Planck, BLAST, and cross-correlated BLAST/ACT power spectrum data over a broad range of frequency $(150 \mathrm{GHz}<v<1200 \mathrm{GHz})$ and angular scale (multipole moment $100<\ell<9000$ ). We find that the clustering power varies with angular scale as $\ell^{-n}$ with $n=1.25 \pm 0.06$ and that the SED of the clustering can be described as a modified blackbody with emissivity index $\beta=2.20 \pm 0.07$ and effective temperature $T_{\text {eff }}=9.7 \mathrm{~K}$. Our work does not rely on any assumptions regarding the physical properties of the IR sources (host halos, redshift distribution, etc.).

As well as providing a simple template for use in CMB foreground subtraction, we have established that the Planck and BLAST/BLAST $\times$ ACT data sets appear compatible when bandpass filters, flux cut, and calibration are accounted for, as described in Sections 2 and 3. We make predictions for the IR clustering power for the Planck, ACT, and SPT CMB bands; our predictions for the ACT and SPT bands at around 150 and $220 \mathrm{GHz}$ are fully consistent with existing measurements (Dunkley et al. 2011; Shirokoff et al. 2011).

There is uncertainty in the Planck shot-noise levels because Planck does not probe small enough scales for the shot noise to become dominant. We find reasonable consistency between the shot-noise levels from our fitting and the predictions of the parametric IR galaxy evolution model of Béthermin et al. (2011). We repeated our fitting using this model's predictions as priors on the Planck shot-noise levels and found that there was minimal effect on the scale or frequency dependence of the clustering power. Number counts obtained via $P(D)$ analysis (probability of deflection-modeling the probability distribution function of observed flux in each map pixel-e.g., Patanchon et al. 2009; Glenn et al. 2010) may be useful for constraining shot-noise levels in future work.

Upcoming data from Herschel, Planck, ACT, SPT, and cross-correlations will allow us to look for any variations in the clustering angular scale dependence with frequency. Understanding this scale dependence in the millimeter CMB bands is important not only for extracting unbiased estimates of cosmological parameters, but also for constraining other components of the measured spectrum, such as the thermal and kinetic SZ effect, and any cross-component correlations, for instance SZ-IR. 
Over the range of angular scales considered we would expect to be probing both the linear and nonlinear clustering regimes. This is supported by the fact that the combined Planck and BLAST data strongly reject a linear bias clustering model (Section 4.1.5). The linear and nonlinear components have different scale dependence, with the linear component dominating on large scales and the nonlinear on small scales. The fact that our study has found that a power-law scale dependence provides a good fit to the Planck and BLAST IR point-source clustering power is not inconsistent with this picture; instead it suggests that the sum of the linear and nonlinear components is sufficiently close to a power law that the current data are unable to reveal any deviations. We can draw a comparison to measurements of the angular correlation function, $w(\theta)$, of luminous red galaxies and galaxies in the main SDSS sample, which have revealed deviations from power-law behavior (e.g., Zehavi et al. 2004, 2005; Blake et al. 2008), whereas earlier studies of galaxy clustering were unable to do so (e.g., Zehavi et al. 2002). We may expect similar deviations to be detected in future IR source power spectra, in particular with Herschel/SPIRE data, which will yield higher quality data than BLAST out to smaller scales with its improved angular resolution.

The correlation function of resolved submillimeter sources (e.g., Cooray et al. 2010; Maddox et al. 2010) provides complementary information to the power spectra of unresolved sources considered in this work. Current data are limited; Guo et al. (2011) measure $\xi(r) \propto r^{-\gamma}$ with $\gamma \sim 2$, corresponding to $C_{\ell}^{\text {clust }} \propto \ell^{-1}$, for low-redshift $(z<0.5)$ Herschel-ATLAS galaxies, although their result is consistent with ours within errors. The full Herschel-ATLAS survey (Eales et al. 2010) will cover 30 times more sky and provide far tighter constraints. Discrepancies between the $C_{\ell}^{\text {clust }}$ and $\xi(r)$ or $w(\theta)$ measurements would indicate differences in clustering properties of the bright submillimeter galaxies compared to the fainter population, and thus both statistics are important for constraining models of galaxy clustering and evolution.

This work was supported by the U.S. National Science Foundation through awards AST-0408698 for the ACT project, and PHY-0355328, AST-0707731, and PIRE-0507768. G.A. is supported by an STFC studentship and funding was also provided by Princeton University and the University of Pennsylvania, RCUK Fellowship (J.D.), ERC grant 259505 (J.D.), and NASA grant NNX08AH30G (A.H.). ACT. operates in the Chajnantor Science Preserve in Northern Chile under the auspices of the Comisión Nacional de Investigación Científica y Tecnológica (CONICYT). Data acquisition electronics were developed with assistance from the Canada Foundation for Innovation. We thank Matthieu Béthermin for discussions about the B11 IR model, Olivier Doré, Guilaine Lagache, and Bill Jones for discussions about the Planck data, Joaquin Vieira for information about SPT filters, and Gaelen Marsden, Bruce Partridge, and George Efstathiou for helpful suggestions.

\section{REFERENCES}

Amblard, A., Cooray, A., Serra, P., et al. 2011, Nature, 470, 510

Béthermin, M., Dole, H., Lagache, G., Le Borgne, D., \& Penin, A. 2011, A\&A, 529, A4

Blain, A. W., Smail, I., Ivison, R. J., \& Kneib, J.-P. 1999, MNRAS, 302, 632
Blake, C., Collister, A., \& Lahav, O. 2008, MNRAS, 385, 1257

Bond, J. R. 1996, in Cosmology and Large Scale Structure, Les Houches Session LX, ed. R. Schaeffer (London: Elsevier), 496

Bond, J. R., Carr, B. J., \& Hogan, C. J. 1986, ApJ, 306, 428

Bond, J. R., Carr, B. J., \& Hogan, C. J. 1991, ApJ, 367, 420

Chapin, E. L., Pope, A., Scott, D., et al. 2009, MNRAS, 398, 1793

Coil, A. L., Newman, J. A., Cooper, M. C., et al. 2006, ApJ, 644, 671

Cooray, A., Amblard, A., Wang, L., et al. 2010, A\&A, 518, L22

Cooray, A., \& Sheth, R. 2002, Phys. Rep., 372, 1

Coppin, K., Halpern, M., Scott, D., et al. 2008, MNRAS, 384, 1597

Das, S., Marriage, T. A., Ade, P. A. R., et al. 2011, ApJ, 729, 62

Draine, B. T. 2003, ARA\&A, 41, 241

Dunkley, J., Bucher, M., Ferreira, P. G., Moodley, K., \& Skordis, C. 2005, MNRAS, 356, 925

Dunkley, J., Hlozek, R., Sievers, J., et al. 2011, ApJ, 739, 52

Eales, S., Dunne, L., Clements, D., et al. 2010, PASP, 122, 499

Fixsen, D. J., Dwek, E., Mather, J. C., Bennett, C. L., \& Shafer, R. A. 1998, ApJ, 508,123

Fowler, J. W., Acquaviva, V., Ade, P. A. R., et al. 2010, ApJ, 722, 1148

Giavalisco, M., Steidel, C. C., Adelberger, K. L., et al. 1998, ApJ, 503, 543

Gispert, R., Lagache, G., \& Puget, J. L. 2000, A\&A, 360, 1

Glenn, J., Conley, A., Béthermin, M., et al. 2010, MNRAS, 409, 109

Guo, Q., Cole, S., Lacey, C. G., et al. 2011, MNRAS, 412, 2277

Haiman, Z., \& Knox, L. 2000, ApJ, 530, 124

Hajian, A., Acquaviva, V., Ade, P. A. R., et al. 2011, ApJ, 740, 86

Hajian, A., Viero, M. P., Addison, G., et al. 2012, ApJ, 744, 40

Hall, N. R., Keisler, R., Knox, L., et al. 2010, ApJ, 718, 632

Hughes, D. H., Serjeant, S., Dunlop, J., et al. 1998, Nature, 394, 241

Hwang, H. S., Serjeant, S., Dunlop, J., et al. 2010, MNRAS, 409, 75

Keisler, R., Reichardt, C. L., Aird, K. A., et al. 2011, ApJ, 743, 28

Knox, L., Cooray, A., Eisenstein, D., \& Haiman, Z. 2001, ApJ, 550, 7

Komatsu, E., Smith, K. M., Dunkley, J., et al. 2011, ApJS, 192, 18

Lagache, G., Abergel, A., Boulanger, F., Désert, F. X., \& Puget, J.-L. 1999, A\&A, 344, 322

Lagache, G., Bavouzet, N., Fernandez-Conde, N., et al. 2007, ApJ, 665, L89

Larson, D., Dunkley, J., Hinshaw, G., et al. 2011, ApJS, 192, 16

Lee, K.-S., Giavalisco, M., Gnedin, O. Y., et al. 2006, ApJ, 642, 63

Maddox, S. J., Dunne, L., Rigby, E., et al. 2010, A\&A, 518, L11

Marsden, G., Ade, P. A. R., Bock, J. J., et al. 2009, ApJ, 707, 1729

Marsden, G., Chapin, E. L., Halpern, M., et al. 2011, MNRAS, 417, 1192

Metropolis, N., Rosenbluth, A. W., Rosenbluth, M. N., \& Teller, A. H. 1953, J. Chem. Phys., 21, 1087

Millea, M., Doré, O., Dudley, J., et al. 2012, ApJ, 746, 4

Negrello, M., Perrotta, F., González-Nuevo, J., et al. 2007, MNRAS, 377, 1557

Ouchi, M., Hamana, T., Shimasaku, K., et al. 2005, ApJ, 635, L117

Pascale, E., Ade, P. A. R., Bock, J. J., et al. 2009, ApJ, 707, 1740

Patanchon, G., et al. 2009, ApJ, 707, 1750

Peacock, J. A., \& Smith, R. E. 2000, MNRAS, 318, 1144

Peebles, P. J. E. 1980, The Large-scale Structure of the Universe (Research Supported by the National Science Foundation, Princeton; NJ: Princeton Univ. Press), 435

Planck Collaboration, et al. 2011a, A\&A, 536, A1

Planck Collaboration, et al. 2011b, A\&A, 536, A18

Planck HFI Core Team, et al. 2011, A\&A, 536, A6

Puget, J.-L., Abergel, A., Bernard, J.-P., et al. 1996, A\&A, 308, L5

Righi, M., Hernández-Monteagudo, C., \& Sunyaev, R. A. 2008, A\&A, 478, 685

Scoccimarro, R., Feldman, H. A., Fry, J. N., \& Frieman, J. A. 2001, ApJ, 546, 652

Scott, D., \& White, M. 1999, A\&A, 346, 1

Sehgal, N., Bode, P., Das, S., et al. 2010, ApJ, 709, 920

Shirokoff, E., Reichardt, C. L., Shaw, L., et al. 2011, ApJ, 736, 61

Silk, J. 1968, ApJ, 151, 459

Sunyaev, R. A., \& Zel'dovich, Y. B. 1970, Ap\&SS, 7, 3

Tegmark, M., Dodelson, S., Eisenstein, D. J., et al. 2002, ApJ, 571, 191

Truch, M. D. P., Ade, P. A. R., Bock, J. J., et al. 2009, ApJ, 707, 1723

Viero, M. P., Ade, P. A. R., Bock, J. J., et al. 2009, ApJ, 707, 1766

Wake, D. A., Whitaker, K. E., Labbé, I., et al. 2011, ApJ, 728, 46

Watson, D. F., Berlind, A. A., \& Zentner, A. R. 2011, ApJ, 738, 22

White, M. J., \& Majumdar, S. 2004, ApJ, 602, 565

Zehavi, I., Blanton, M. R., Frieman, J. A., et al. 2002, ApJ, 571, 172

Zehavi, I., Weinberg, D. H., Zheng, Z., et al. 2004, ApJ, 608, 16

Zehavi, I., Zheng, Z., Weinberg, D. H., et al. 2005, ApJ, 630, 1 